Brit. J. Psychiat. (1983), 143, 201-206

\title{
Correspondence
}

Correspondents should note that space is limited and shorter letters have a greater chance of publication. The Editors reserve the right to cut letters and also to eliminate multitudinous references. Please try to be concise, strictly relevant and interesting to the reader, and check the accuracy of all references in Journal style.

\section{DEAD SOULS: CRISIS INTERVENTION IN GERIATRICS}

DeAr SIR,

I am grateful for this opportunity to reply to Grundy and Arie's criticism of my paper (Journal, March 1983, 142, 312-13). They have commenced the task for me by replying to some of the points they raise. For instance, they begin by suggesting that our claim to practice psycho-geriatrics with a lower mortality rate is untrue and then go on to conclude that our survival rate is indeed better. They assume (erroneously) that we operate a 'no admission' policy and then go on to prove that our admission rate is the same elsewhere. The central fallacy of their criticism is, however, that they mistakenly assume that this is a comparative study. We had replicated Sainsbury's methodology (with additions and modifications), and we listed his figures in the tables as a historical perspective and not as a statistical comparison. We did not perform any comparative tests and when we make comparative remarks it is in a general discussion in relation to more recent studies, including Arie's.

As any qualified statistician knows, valid comparisons cannot be made between two populations as different as Barnet and Chichester, particularly as the samples are nearly two decades apart in time. The elegent statistical arguments of Arie and Grundy are fundamentally invalid because the samples are not comparable.

Grundy and Arie's suggestion that referrals may be discouraged from our service has no basis in fact, because we show the highest referral rate in any published study and twice the referral rate to Arie's service in Goodmayes. Their allegation that there is a leakage to Social Services is unclear because the social worker is an integral part of the crisis service. The referral rate to the geriatrician has not risen since our policy was launched. They compare us, emotively, with anti-abortionists who deny the problem and evade it. We know of no anti-abortionist who is willing to go out to the patients at home at any time of the day or night within two hours of referral seven days a week.
The correspondents, by adding up the admissions to mental hospital and Part III, claim that our admission rate is the same as elsewhere and that this invalidates our argument. On the contrary, if we are operating strategies that achieve the same result at a much lower cost, then we have policies that demand to be examined, particularly in these times of cut backs.

Placement in Part III does not involve the labelling or the cost of mental hospitalization, and we achieved this despite the exceptionally long waiting lists in Barnet which has the lowest figure for Part III beds of all the London Boroughs.

Grundy and Arie argue that our low mortality is due to improved life expectancy. If this were true it is curious that this factor did not affect the other studies we list in our discussion of this issue, including Arie's, which despite having been conducted only seven years earlier experienced twice our mortality rate. They claim that there is no evidence that admission is related to mortality when, in fact, we list several studies that suggest this conclusion.

Arie has long been a fighter for more resources and we fully support him in this battle, but in the meantime we also need to look at methods of optimising the few we have in order to get the maximum use out of them. We believe the policies we have initiated are a step in this direction and the fact that they have remained operationally viable over the last eight years-a period in which our referral rate has doubled, whilst our admission beds have been cut by 60 per cent-is practical evidence of the clinical usefulness of our methods. Our crisis service has all the virtues of availability, quickness of response, multi-disciplinary assessment, and continuity of care, that Arie has for so long preached. I would have thought that making such services available round the clock is a development to be welcomed.

Barnet General Hospital,

L. RATNA

Barnet,

Hertfordshire EN5 3DJ 